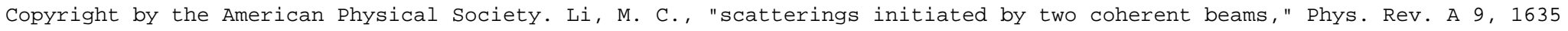
(1974); DOI: http://dx.doi.org/10.1103/PhysRevA.9.1635

\title{
Scatterings initiated by two coherent beams
}

\author{
Ming Chiang Li \\ Department of Physics, Virginia Polytechnic Institute and State University, Blacksburg, Virginia 24061
} (Received 9 October 1973)

\begin{abstract}
A theory, which exhibits the characteristic features of scatterings initiated by two coherent beams, is discussed in the present paper. We show that the imaginary part of the scattering amplitude is an experimentally measurable quantity. To know the imaginary part of the amplitude is quite important for certain aspects of quantum mechanics, for example, the dispersion relation for a fixed momentum transfer. This relation has not yet been experimentally verified.
\end{abstract}

\section{INTRODUCTION}

The subject of this paper is scattering initiated by two coherent beams. We are quite familiar with coherent beams of light. They are formed through the division of the light from a source by a suitable apparatus, i.e., two pinholes in Young's experiment, Fresnel's mirrors and biprism, ${ }^{1}$ etc. When they are superposed, the intensity in the region of superposition is found to vary from point to point between maxima which exceed the sum of the intensities in the beams, and minima which may be zero. This phenomenon is called interference. From the interference pattern we can obtain the wavelength of the beams. Coherent beams for electrons are obtained in much the same way. The primary electron beam is split by an apparatus such as the Marton-type ${ }^{2}$ interferometer (a rough equivalent of the MachZehnder-type optical interferometer), or a Mollenstedt-Duker interferometer ${ }^{3}$ (the electron-optical analog of Fresnel's biprism). Electron interferences up to an order of 300 wavelength differences have been observed. The interference fringes are very luminous, show strong contrast, and are steady. ${ }^{4}$

Historically, real-interference phenomena have played a key role in establishing the wave nature of light, and today are of great practical importance, in spectroscopy and metrology, for example. The same is not true for the electron. The wave nature of the electron was established through electron diffraction experiments, in which only one beam is observed at any point of the receptor, and interference is between the elementary wavelets. Why the electron-interference experiments have played such a minor role lies in the associated experimental difficulties. To obtain reasonable penetration into the diffractor, one has to work at electron energies of the order of $50 \mathrm{keV} .^{4}$ At this energy, the electron wavelength is only about $0.05 \AA$.
This short wavelength means that the apparatus of slits, point source, etc., must be scaled down by a factor of almost 100 from those of light optics. It also means that the observed fringe spacing is considerably less than $\frac{1}{10}$ of a light optical one. Thus, the difficulty of electron realinterference experiments is several orders of magnitude beyond those of light.

Interference between the primary and scatter ed beams is a real interference of a different type..$^{5}$ It enables us to determine the phase of the scattered beam, by comparison with a coherent background. The resultant diffraction pattern is called a hologram. ${ }^{1}$ With the introduction of laser beams, the hologram technique has attained wide usage in light optics. Due to the small cross-sectional area of primary incident beams in the electron scattering, the primary and scattered beams extinguish one another outside a very small angle. Except in the forward direction, the interference between them is not present. The primary beam is much stronger than the scattered one. If there exists a mechanism to make the interference possible, the primary beam has to attenuate first. This would complicate the issue. In practice, the amplitude (not the phase) is the only measurable quantity in electron scattering.

The scattering initiated by two coherent beams is an interesting one. Such beams, as we have seen, are obtainable for both light and electrons. The beams intersect at the target with a finite intersection angle. The two scattered outgoing waves interfere with one another along the same radial direction. The resultant effects are recorded by the receptor. Insofar as the interference is concerned, it differs from the abovementioned real interferences, in which the beams are in different directions.

We will show that from the experimentally observed coherent cross section one is able to determine the phase (in addition to the magnitude) 
of the scattering amplitude. This is the intended result in the direct interference of the primary and scattered beams, but in electron scattering has not been obtained experimentally.

We will show that from the experimentally observed coherent differential cross section one is able to obtain the relative phase between the initial coherent beams. The scatterer here plays the role of the analyzer. Since the electron-beam wavelength can be determined by the accelerating voltage, the scattering may be treated as an electron interference experiment. An interesting point should be mentioned. In these experiments, measurements of interference effects are obtained from differential cross sections and not from the observation of fringe effects. This eases the monoenergetic restrictions and size limitations on the incident electron beams.

In Sec. II, we discuss the general theory of coherent scattering. We study the scattering by a spherical potential in Sec. III. The measurement of the "relative factor" between the initial coherent beams is also given there. In Sec. IV, spheroidal potentials, which have important applications in scattering by molecules and deformed nuclei are discussed. The coherent scattering by a spin-dependent potential is worked out in Sec. V. Some comments are contained in Sec. VI.

\section{COHERENT SCATTERING}

In the coherent scattering we use two incident coherent beams. In the conventional scattering only one incident beam is used. What do we mean by coherent beams? It means that when these two beams are reunited at the photographic plate or other type of receptor, one observes interference phenomena. The coherent light rays, which are the coherent beams of photon particles, are well known. The coherent electron beams can also be obtained through the splitting of a primary beam as in electron interferometers by Marton and by Mollenstedt and Duker. The coherent beams for other elementary particles, although difficult, in principle still can be constructed. The two coherent beams may be different in direction, but must have the same energy Let these two beams have the momenta $\vec{k}_{1}$ and $\vec{k}_{2}$, then

$$
\overrightarrow{\mathrm{k}}_{1}^{2}=\overrightarrow{\mathrm{k}}_{2}^{2}=2 m E / \hbar^{2},
$$

where $E$ is the energy of the beams and $m$ is the mass of the particles. The incident beams can be described by the wave function ${ }^{5}$

$$
e^{i \overrightarrow{\mathrm{K}}_{1} \cdot \overrightarrow{\mathrm{r}}}+a e^{i \overrightarrow{\mathrm{K}}_{2} \cdot \overrightarrow{\mathrm{r}}},
$$

where $a$ denotes the relative phase and amplitude of these two waves. In the collision process the coherent waves are changed and satisfy the Schrödinger equation

$$
-\left(\hbar^{2} / 2 m\right) \nabla^{2} \Psi(\overrightarrow{\mathbf{r}})+V(\overrightarrow{\mathbf{r}}) \Psi(\overrightarrow{\mathbf{r}})=E \Psi(\overrightarrow{\mathbf{r}}),
$$

and the asymptotic condition

$$
\Psi(\overrightarrow{\mathrm{r}}) \underset{r \rightarrow \infty}{\sim} e^{i \overrightarrow{\mathrm{k}}_{1} \cdot \overrightarrow{\mathrm{r}}}+a e^{i \overrightarrow{\mathrm{k}}_{2} \cdot \overrightarrow{\mathrm{r}}}+F\left(\overrightarrow{\mathrm{k}}_{1}, \overrightarrow{\mathrm{k}}_{2} ; \overrightarrow{\mathrm{n}}\right) e^{i k r} / r
$$

where $V(\overrightarrow{\mathbf{r}})$ is the potential of a fixed center. For simplicity, it is taken as a short-range potential. $\overrightarrow{\mathrm{n}}$ is the direction of the scattered wave, which comes from the disturbance of the incident waves by the scattered potential. $F\left(\overrightarrow{\mathrm{k}}_{1}, \overrightarrow{\mathrm{k}}_{2} ; \overrightarrow{\mathrm{n}}\right)$ is the scattering amplitude and is related to the experimentally observed differential cross section

$$
d \Sigma\left(\overrightarrow{\mathrm{k}}_{1}, \overrightarrow{\mathrm{k}}_{2} ; \vec{n}\right)=\left(1+|a|^{2}\right)^{-1}\left|F\left(\overrightarrow{\mathrm{k}}_{1}, \overrightarrow{\mathrm{k}}_{2} ; \overrightarrow{\mathrm{n}}\right)\right|^{2} d \Omega_{\overrightarrow{\mathrm{n}}},
$$

where $d \Omega_{\mathrm{n}}$ is the solid angle. Equation (2.3)-(2.5) form the theoretical foundation of the coherent scattering.

We shall begin to solve the coherent-scattering problem. It is observed that the Schrödinger equation (2.3) and the incident wave in Eq. (2.4) are both linear; therefore, the wave function $\Psi(\overrightarrow{\mathrm{r}})$ can be expressed as

$$
\Psi(\overrightarrow{\mathrm{r}})=\psi_{1}(\overrightarrow{\mathrm{r}})+a \psi_{2}(\overrightarrow{\mathrm{r}}),
$$

where wave functions $\psi_{1}(\overrightarrow{\mathbf{r}})$ and $\psi_{2}(\overrightarrow{\mathbf{r}})$ satisfy the Schrödinger equations

$$
-\left(\hbar^{2} / 2 m\right) \nabla^{2} \psi_{i}(\overrightarrow{\mathbf{r}})+V(\overrightarrow{\mathrm{r}}) \psi_{i}(\overrightarrow{\mathrm{r}})=E \psi_{\boldsymbol{i}}(\overrightarrow{\mathrm{r}}), \quad i=1,2
$$

and the asymptotic conditions

$$
\psi_{i}(\overrightarrow{\mathrm{r}}) \underset{r \rightarrow \infty}{\sim} e^{i \overrightarrow{\mathrm{k}}_{i} \cdot \overrightarrow{\mathrm{r}}}+f\left(\overrightarrow{\mathrm{k}}_{i} ; \overrightarrow{\mathrm{n}}\right) e^{i k r} / r, \quad i=1,2 .
$$

Equation $\left(2.3^{\prime}\right)$ and $\left(2.4^{\prime}\right)$ are actually the Schrödinger equation and asymptotic conditions of the conventional non-coherent-scattering problem, in which the incident beam has momentum $\overrightarrow{\mathrm{k}}_{i}$ and yields a scattering amplitude $f\left(\overrightarrow{\mathrm{k}}_{i} ; \overrightarrow{\mathrm{n}}\right)$. The coherent-scattering amplitude may be expressed as

$$
F\left(\overrightarrow{\mathrm{k}}_{1}, \overrightarrow{\mathrm{k}}_{2} ; \overrightarrow{\mathrm{n}}\right)=f\left(\overrightarrow{\mathrm{k}}_{1} ; \overrightarrow{\mathrm{n}}\right)+a f\left(\overrightarrow{\mathrm{k}}_{2} ; \overrightarrow{\mathrm{n}}\right) .
$$

The conventional differential cross section has the form

$$
d \sigma\left(\overrightarrow{\mathrm{k}}_{i} ; \overrightarrow{\mathrm{n}}\right)=\left|f\left(\overrightarrow{\mathrm{k}}_{i} ; \overrightarrow{\mathrm{n}}\right)\right|^{2} d \Omega_{\mathrm{n}} .
$$

From Eqs. (2.5), (2.7), and (2.8) we obtain the relationship between the coherent and conventional differential cross sections: 


$$
\begin{aligned}
d \Sigma\left(\overrightarrow{\mathrm{k}}_{1}, \overrightarrow{\mathrm{k}}_{2} ; \overrightarrow{\mathrm{n}}\right) & \\
=\left(1+|a|^{2}\right)^{-1}\{ & d \sigma\left(\overrightarrow{\mathrm{k}}_{1} ; \overrightarrow{\mathrm{n}}\right)+|a|^{2} d \sigma\left(\overrightarrow{\mathrm{k}}_{2} ; \overrightarrow{\mathrm{n}}\right) \\
+ & \left.2 \operatorname{Re}\left[a f^{*}\left(\overrightarrow{\mathrm{k}}_{1} ; \overrightarrow{\mathrm{n}}\right) f\left(\overrightarrow{\mathrm{k}}_{2} ; \overrightarrow{\mathrm{n}}\right)\right] d \Omega_{\mathrm{n}}\right\} .
\end{aligned}
$$

Equation (2.9) indicates that in the coherent scattering the observed differential cross section in Eq. (2.5) contains the interference effect of the conventional scattering amplitude. This effect provides us a direct way to measure the real and imaginary parts of the scattering amplitude. However, in the conventional scattering only its magnitude is measured. For elastic scattering the conservation of the number of the particles leads to a restriction on the scattering amplitude ${ }^{6}$

$$
f\left(\overrightarrow{\mathrm{k}}_{i} ; \overrightarrow{\mathrm{n}}\right)-f *\left(\overrightarrow{\mathrm{k}} ; \overrightarrow{\mathrm{n}}_{i}\right)=\frac{i k}{2 \pi} \int f\left(\overrightarrow{\mathrm{k}}_{i} ; \overrightarrow{\mathrm{n}}^{\prime}\right) f *\left(\overrightarrow{\mathrm{k}} ; \overrightarrow{\mathrm{n}}^{\prime}\right) d \Omega_{\overrightarrow{\mathrm{n}}^{\prime}},
$$

where

$$
\overrightarrow{\mathrm{k}}=k \overrightarrow{\mathrm{n}}, \quad \overrightarrow{\mathrm{n}}_{i}=\overrightarrow{\mathrm{k}}_{\boldsymbol{i}} / k
$$

The relation in Eq. (2.10) is also called the unitarity relation of the scattering amplitude. From Eqs. (2.9) and (2.10) the total coherent cross section has the form

$$
\begin{aligned}
\Sigma\left(\overrightarrow{\mathrm{k}}_{1}, \overrightarrow{\mathrm{k}}_{2}\right)= & \int d \Sigma\left(\overrightarrow{\mathrm{k}}_{1}, \overrightarrow{\mathrm{k}}_{2} ; \overrightarrow{\mathrm{n}}\right)=\frac{\sigma\left(k_{1}\right)}{1+|a|^{2}}+\frac{|a|^{2} \sigma\left(k_{2}\right)}{1+|a|^{2}} \\
& +\frac{4 \pi}{1+|a|^{2}} \operatorname{Re}\left(\frac{a}{i k}\left[f\left(\overrightarrow{\mathrm{k}}_{2} ; \overrightarrow{\mathrm{n}}_{1}\right)-f^{*}\left(\overrightarrow{\mathrm{k}}_{1} ; \overrightarrow{\mathrm{n}}_{2}\right)\right]\right),
\end{aligned}
$$

where

$$
\sigma\left(\overrightarrow{\mathrm{k}}_{i}\right)=\int d \sigma\left(\overrightarrow{\mathrm{k}}_{i}, \overrightarrow{\mathrm{n}}\right)=\int\left|f\left(\overrightarrow{\mathrm{k}}_{i}, \overrightarrow{\mathrm{n}}\right)\right|^{2} d \Omega_{\overrightarrow{\mathrm{n}}}
$$

is the conventional total cross section. For $\vec{k}_{1}$ $=\vec{k}_{2}$, the two coherent beams reduce to a single beam, and Eq. (2.12) becomes

$$
\Sigma\left(\overrightarrow{\mathrm{k}}_{1}, \overrightarrow{\mathrm{k}}_{1}\right)=\sigma\left(\overrightarrow{\mathrm{k}}_{1}\right)+(8 \pi / k)\left(1+|a|^{2}\right)^{-1} \operatorname{Re} a \operatorname{Im} f\left(\overrightarrow{\mathrm{k}}_{1} ; \overrightarrow{\mathrm{n}}\right) .
$$

The scattering amplitude satisfies the optical theorem

$$
\operatorname{Im} f\left(\overrightarrow{\mathrm{k}}_{1}, \overrightarrow{\mathrm{n}}_{1}\right)=(k / 4 \pi) \sigma\left(\overrightarrow{\mathrm{k}}_{1}\right) .
$$

Then we have

$$
\Sigma\left(\overrightarrow{\mathrm{k}}_{1} ; \overrightarrow{\mathrm{k}}_{1}\right)=\sigma\left(\overrightarrow{\mathrm{k}}_{1}\right)\left(1+\frac{2 \mathrm{Re} a}{1+|a|^{2}}\right) .
$$

The factor in the above equation comes from the difference between the incident beam fluxes in the coherent and conventional scatterings. Before scattering, the coherent beams extinguish one another, the total incident flux is the independent sum

$$
\left(1+|a|^{2}\right) \hbar \boldsymbol{k} / 2 m .
$$

If two coherent beams reduce to a single beam, the above flux definition is not appropriate and the corrected one should be

$$
|1+a|^{2} \hbar k / 2 m \text {. }
$$

These differences have not been reflected in Eq. (2.16). Nevertheless when the correct incident flux is used, Eq. (2.16) gives the expected result. We may say that the conventional scattering is a special case of the coherent scattering.

We have studied the general feature of coherent scattering by a fixed potential. We may ask whether the same scattering is also useful in a twobody scattering problem. A careful study reveals that the coherent incident beams do not give rise to a coherent scattering in this problem. The main reason is not difficult to explain. In the coherent beams, the incident particle can be in one of the two momenta $\vec{p}_{1}$ and $\vec{p}_{2}$ state,

$$
\left|\overrightarrow{\mathrm{p}}_{1}\right\rangle+a\left|\overrightarrow{\mathrm{p}}_{2}\right\rangle \text {. }
$$

But other particles are in the pure single-momentum states. The momentum conservation, which does not appear in the fixed potential scattering, eliminates the cross term of the coherent cross section. Then the experimentally observed quantity does not contain any information of the coherent effects in the two-body reaction.

\section{SPHERICAL POTENTIAL}

In Sec. II, a general theory of the coherent potential scattering was studied. In further study we will be more specific on the form of the potential. At the present the spherical potential is considered. In this case the amplitude $f\left(\overrightarrow{\mathrm{k}}_{i} ; \overrightarrow{\mathrm{n}}\right)$ and differential cross section $d \sigma\left(\overrightarrow{\mathrm{k}}_{i} ; \overrightarrow{\mathrm{n}}\right)$ in Eq. (2.8) depend only on the angle between $\vec{n}_{i}$ and $\vec{n}$ :

$$
f\left(\overrightarrow{\mathrm{k}}_{i} \cdot \overrightarrow{\mathrm{n}}\right)=f_{k}\left(\overrightarrow{\mathrm{n}}_{i} \cdot \overrightarrow{\mathrm{n}}\right)
$$

and

$$
d \sigma\left(\overrightarrow{\mathrm{k}}_{\boldsymbol{i}} \cdot \overrightarrow{\mathrm{n}}\right)=d \sigma_{k}\left(\overrightarrow{\mathrm{n}}_{i} \cdot \overrightarrow{\mathrm{n}}\right) .
$$

Then the coherent cross section in Eq. (2.12) may be rewritten

$$
\begin{aligned}
\Sigma\left(\overrightarrow{\mathrm{k}}_{1}, \overrightarrow{\mathrm{r}}_{2}\right)= & \sigma(k)+(8 \pi / k)\left(1+|a|^{2}\right)^{-1} \\
& \times \operatorname{Re} a \operatorname{Im} f_{k}\left(\overrightarrow{\mathrm{n}}_{1} \cdot \overrightarrow{\mathrm{n}}_{2}\right) .
\end{aligned}
$$

Equation (3.3) expresses the fact that the coherent cross section is related to the imaginary part 
of the scattering amplitude. The dependence on the factor $a$ in Eq. (3.3) can be evaluated through a simple consideration. The spherical differential cross section in Eq. (2.9) at $\vec{n}_{1} \cdot \overrightarrow{\mathrm{n}}=\overrightarrow{\mathrm{n}}_{2} \cdot \overrightarrow{\mathrm{n}}$ has the form

$$
d \Sigma\left(\overrightarrow{\mathrm{k}}_{1}, \overrightarrow{\mathrm{k}}_{2} ; \overrightarrow{\mathrm{n}}\right)=\left(1+\frac{2 \mathrm{Re} a}{1+|a|^{2}}\right) d \sigma_{k}\left(\overrightarrow{\mathrm{n}}_{1} \cdot \overrightarrow{\mathrm{n}}\right) .
$$

In arriving at Eq. (3.4), we have utilized Eqs. (2.8), (3.1), and (3.2). The imaginary part of the scattering amplitude in Eq. (3.3) can be expressed finally in terms of experimentally measured quantities

$$
\begin{aligned}
\operatorname{Im} f_{k}\left(\overrightarrow{\mathrm{n}}_{1} \cdot \overrightarrow{\mathrm{n}}_{2}\right)= & \frac{k}{4 \pi}\left[\Sigma\left(\overrightarrow{\mathrm{k}}_{1}, \overrightarrow{\mathrm{k}}_{2}\right)-\sigma(k)\right] \\
& \times\left(\frac{d \Sigma\left(\overrightarrow{\mathrm{k}}_{1}, \overrightarrow{\mathrm{k}}_{2} ; \overrightarrow{\mathrm{n}}\right)}{d \sigma_{k}\left(\overrightarrow{\mathrm{n}}_{1} \cdot \overrightarrow{\mathrm{n}}\right)}-1\right)^{-1} .
\end{aligned}
$$

In the conventional sense, this is not a directly measurable quantity. Hence the coherent scattering has opened a new chapter in the experimental determination of the scattering amplitude.

In reaching Eq. (3.5) we have assumed that only the elastic process is occurring in the scattering. However, in the elastic scattering some inelastic processes might be present. To be more general we shall study the effect of these processes. This effect can be understood through a consideration of the partial-wave representation of the scattering amplitude:

$$
f_{k}\left(\overrightarrow{\mathrm{n}}_{i} \cdot \overrightarrow{\mathrm{n}}\right)=\frac{2 \pi}{i k} \sum_{i=0}^{\infty} \sum_{m=-l}^{l}\left[S_{l}(k)-1\right] Y_{l m}\left(\overrightarrow{\mathrm{n}}_{i}\right) Y_{l m}^{*}(\overrightarrow{\mathrm{n}}),
$$

where

$$
S_{l}(k)=e^{2 i \delta_{l}(k)}
$$

and $Y_{l m}(\vec{n})$ is a spherical harmonic. In the presence of the inelastic process, the phase shift $\delta_{l}(k)$ is complex. From Eq. (2.7), the coherentscattering amplitude is a linear superposition of the conventional scattering amplitudes, then its partial wave expansion has the form

$$
\begin{aligned}
F\left(\overrightarrow{\mathrm{k}}_{1}, \overrightarrow{\mathrm{k}}_{2} ; \overrightarrow{\mathrm{n}}\right)= & \frac{2 \pi}{i k} \sum_{l=0}^{l} \sum_{m=-l}^{l}\left[S_{l}(k)-1\right] \\
& \times Y_{l m}^{*}(\overrightarrow{\mathrm{n}})\left[Y_{l m}\left(\overrightarrow{\mathrm{n}}_{1}\right)+a Y_{l m}\left(\overrightarrow{\mathrm{n}}_{2}\right)\right] .
\end{aligned}
$$

With the help of the orthogonality relations between the spherical harmonics and the addition theorem

$$
P_{l}\left(\overrightarrow{\mathrm{n}}_{1} \cdot \overrightarrow{\mathrm{n}}_{2}\right)=\frac{4 \pi}{2 l+1} \sum_{m=-l}^{l} Y_{l m}\left(\overrightarrow{\mathrm{n}}_{1}\right) Y_{l m}^{*}\left(\overrightarrow{\mathrm{n}}_{2}\right)
$$

the total elastic cross section of the coherent scattering might be written

$$
\begin{aligned}
\sum_{e}\left(\overrightarrow{\mathrm{k}}_{1}, \overrightarrow{\mathrm{k}}_{2}\right)= & \left(1+|a|^{2}\right)^{-1} \int\left|F\left(\overrightarrow{\mathrm{k}}_{1}, \overrightarrow{\mathrm{k}}_{2} ; \overrightarrow{\mathrm{n}}\right)\right|^{2} d \Omega \overrightarrow{\mathrm{n}} \\
= & \frac{\pi}{k^{2}}\left(1+|a|^{2}\right)^{-1} \sum_{i=0}^{\infty}(2 l+1)\left|S_{l}(k)-1\right|^{2} \\
& \times\left[\left(1+|a|^{2}\right)+2 P_{l}\left(\overrightarrow{\mathrm{n}}_{1} \cdot \overrightarrow{\mathrm{n}}_{2}\right) \operatorname{Re} a\right]
\end{aligned}
$$

The absorption cross section, which describes the existence of inelastic processes, has the form ${ }^{6}$

$$
\begin{aligned}
\Sigma_{\text {abs }}\left(\overrightarrow{\mathrm{k}}_{1}, \overrightarrow{\mathrm{k}}_{2}\right)= & -\frac{m}{\hbar k}\left(1+|a|^{2}\right)^{-1} \frac{\hbar}{2 i m} \\
& \times \lim _{r \rightarrow \infty} \int[\psi *(\overrightarrow{\mathrm{r}}) \vec{\nabla} \psi(\overrightarrow{\mathrm{r}})-(\vec{\nabla} \psi(\overrightarrow{\mathrm{r}})) * \psi(\overrightarrow{\mathrm{r}})] \cdot d \overrightarrow{\mathrm{A}},
\end{aligned}
$$

where the surface $A$ is a large sphere of radius $r$. In terms of complex phase shifts, the asymptotic form of the coherent wave function $\psi(\overrightarrow{\mathbf{r}})$ in Eq. (2.4) may be expressed as

$$
\begin{aligned}
\psi(\overrightarrow{\mathrm{r}}) \underset{r \rightarrow \infty}{\sim} & \sum_{i=0}^{\infty} \sum_{m=-l}^{l} 4 \pi i^{l}(k r)^{-1} e^{i \delta_{l}(k)} \\
& \times \sin \left[\mathrm{kr}-\frac{1}{2} l \pi+\delta_{l}(k)\right] Y_{l m}^{*}(\overrightarrow{\mathrm{n}}) \\
& \times\left[Y_{l m}^{*}\left(\overrightarrow{\mathrm{n}}_{1}\right)+a Y_{l m}\left(\overrightarrow{\mathrm{n}}_{2}\right)\right]
\end{aligned}
$$

Substituting this into Eq. (3.11) and carrying the integration one obtains

$$
\begin{aligned}
\sum_{\mathrm{abs}}\left(\vec{k}_{1}, \overrightarrow{\mathrm{k}}_{2}\right)= & \frac{8 \pi^{2}}{k^{2}}\left(1+|a|^{2}\right)^{-1} \sum_{l=0}^{\infty} \sum_{m=-l}^{l} \sinh 2 \beta_{l}(k) \\
& \times e^{-2 \beta_{l}(k)}\left|Y_{l m}\left(\overrightarrow{\mathrm{n}}_{1}\right)+a Y_{l m}\left(\overrightarrow{\mathrm{n}}_{2}\right)\right|^{2} \\
= & \frac{\pi}{k^{2}}\left(1+|a|^{2}\right)^{-1} \sum_{l=0}^{\infty}(2 l+1)\left(1-\left|S_{l}(k)\right|^{2}\right) \\
& \times\left[\left(1+|a|^{2}\right)+2 P_{l}\left(\overrightarrow{\mathrm{n}}_{1} \cdot \overrightarrow{\mathrm{n}}_{2}\right) \operatorname{Re} a\right], \quad(3.13)
\end{aligned}
$$

where

$$
\beta_{l}(k)=\operatorname{Im} \delta_{l}(k) .
$$

In arriving at the final form of Eq. (3.13) the addition theorem in Eq. (3.9) is also used. From Eqs. (3.10) and (3.13) the total cross section, which is the sum of the total elastic and absorption cross sections, can be expressed as 


$$
\begin{aligned}
\sum_{\text {tot }}\left(\overrightarrow{\mathrm{k}}_{1}, \overrightarrow{\mathrm{k}}_{2}\right) & =\Sigma_{e}\left(\overrightarrow{\mathrm{k}}_{1}, \overrightarrow{\mathrm{k}}_{2}\right)+\sum_{\mathrm{abs}}\left(\overrightarrow{\mathrm{k}}_{1}, \overrightarrow{\mathrm{k}}_{2}\right) \\
& =\frac{2 \pi}{k^{2}}\left(1+|a|^{2}\right)^{-1} \sum_{l=0}^{\infty}(2 l+1)\left[1-\operatorname{Re} S_{l}(k)\right] \\
& \times\left[\left(1+|a|^{2}\right)+2 P_{l}\left(\overrightarrow{\mathrm{n}}_{1} \cdot \overrightarrow{\mathrm{n}}_{2}\right) \operatorname{Re} a\right] .
\end{aligned}
$$

If $a=0$, we obtain the total cross section for conventional scattering

$$
\sigma_{\mathrm{tot}}(k)=\frac{\pi}{k^{2}} \sum_{l=0}^{\infty}(2 l+1)\left[1-\operatorname{ReS}_{l}(k)\right] .
$$

The imaginary part of the scattering amplitude in Eq. (3.6) is

$$
\operatorname{Im} f_{k}\left(\overrightarrow{\mathrm{n}}_{i} \cdot \overrightarrow{\mathrm{n}}\right)=\frac{1}{2 k} \sum_{i=0}^{\infty}(2 l+1)\left[1-\operatorname{ReS}_{l}(k)\right] P_{l}\left(\overrightarrow{\mathrm{n}}_{i} \cdot \overrightarrow{\mathrm{n}}\right) .
$$

From Eqs. (3.15) - (3.17) we find a relation

$$
\begin{aligned}
\Sigma_{\text {tot }}\left(\overrightarrow{\mathrm{k}}_{1}, \overrightarrow{\mathrm{k}}_{2}\right)= & \sigma_{\text {tot }}(k)+\frac{8 \pi}{k}\left(1+|a|^{2}\right)^{-1} \\
& \times \operatorname{Re} a \operatorname{Im} f_{k}\left(\overrightarrow{\mathrm{n}}_{1} \cdot \overrightarrow{\mathrm{n}}_{2}\right) .
\end{aligned}
$$

This relation is similar to the one in Eq. (3.3), which holds in the absence of the inelastic processes. Following the same steps for deriving Eq. (3.5), a very similar equation might be obtained:

$$
\begin{aligned}
\operatorname{Im} f_{k}\left(\overrightarrow{\mathrm{n}}_{1} \circ \overrightarrow{\mathrm{n}}_{2}\right)= & \frac{k}{4 \pi}\left[\Sigma_{\text {tot }}\left(\overrightarrow{\mathrm{k}}_{1}, \overrightarrow{\mathrm{k}}_{2}\right)-\sigma_{\text {tot }}(k)\right] \\
& \times\left(\frac{d \Sigma^{e}\left(\overrightarrow{\mathrm{k}}_{1}, \overrightarrow{\mathrm{k}}_{2}, \overrightarrow{\mathrm{n}}\right)}{d \sigma_{k}^{e}\left(\overrightarrow{\mathrm{n}}_{1} \cdot \overrightarrow{\mathrm{n}}\right)}-1\right)^{-1},
\end{aligned}
$$

where $d \Sigma^{e}\left(\overrightarrow{\mathrm{k}}_{1}, \overrightarrow{\mathrm{k}}_{2} ; \overrightarrow{\mathrm{n}}\right)$ and $d \sigma_{k}^{e}\left(\overrightarrow{\mathrm{n}}_{1} \cdot \overrightarrow{\mathrm{n}}\right)$ are the coherent and conventional differential cross sections for the elastic process. Now we have achieved our goal of expressing the imaginary part of the scattering amplitude in the presence of inelastic processes in terms of experimentally measurable quantities.

\section{SPHEROIDAL POTENTIAL}

In this section the coherent scattering by a fixed single nonspherical potential is studied. In general the scattering by a nonspherical potential is a complicated subject. It is associated with nonconservation of angular momentum. This conservation is an essential tool for the reduction of the scattering amplitude and a lack of it will lead to a troublesome description of the scattering processes. Here we will take a simple non- spheroidal potential which is spheroidally symmetric. Furthermore, the chosen potential has practical importance in molecular and nuclear physics. A large number of nuclei and molecules are not spherical, but rather spheroidal. These are deformed nuclei and polar molecules. ${ }^{7}$ The potential associated with them is clearly spheroidal. ${ }^{8}$ The cohererit scattering of such systems should have considerable interest.

The spheroidal system has a characteristic direction. We shall call this direction the orientation of the system and denote it by $\vec{\chi}$. For example this might be the direction of the dipole moment for the polar molecules. It is clear that the spheroidal elastic scattering amplitude depends on the orientation $\vec{\chi}$. Meanwhile the amplitude is a function of the incident momentum $\vec{k}_{i}$, the scattered direction $\overrightarrow{\mathrm{n}}$. To express more fully we write the amplitude $f\left(\overrightarrow{\mathrm{k}}_{i} ; \overrightarrow{\mathrm{n}}\right)$ in Eq. (2.7) as $f\left(\overrightarrow{\mathrm{k}}_{i} ; \overrightarrow{\mathrm{n}} ; \vec{\chi}\right)$ in the case of spheroidal scattering. The corresponding coherent scattering amplitude has the form

$$
F\left(\overrightarrow{\mathrm{k}}_{1}, \overrightarrow{\mathrm{k}}_{2} ; \overrightarrow{\mathrm{n}} ; \vec{\chi}\right)=f\left(\overrightarrow{\mathrm{k}}_{1} ; \overrightarrow{\mathrm{n}} ; \vec{\chi}\right)+a f\left(\overrightarrow{\mathrm{k}}_{2} ; \overrightarrow{\mathrm{n}} ; \vec{\chi}\right)
$$

The coherent cross section in Eq. (2.12) is written

$$
\begin{aligned}
\Sigma\left(\overrightarrow{\mathrm{k}}_{1}, \overrightarrow{\mathrm{k}}_{2} ; \vec{\chi}\right)= & \frac{4 \pi}{1+|a|^{2}} \\
& \times \operatorname{Re}\left(\frac{a}{i k}\left[f\left(\overrightarrow{\mathrm{k}}_{2} ; \overrightarrow{\mathrm{n}}_{1} ; \vec{\chi}\right)-f *\left(\overrightarrow{\mathrm{k}}_{1} ; \overrightarrow{\mathrm{n}}_{2} ; \vec{\chi}\right)\right]\right) \\
& +\left(1+|a|^{2}\right)^{-1}\left[\sigma\left(\overrightarrow{\mathrm{k}}_{1} ; \vec{\chi}\right)+\sigma\left(\overrightarrow{\mathrm{k}}_{2}, \vec{\chi}\right)\right], \quad
\end{aligned}
$$

where

$$
\sigma\left(\overrightarrow{\mathrm{k}}_{i} ; \vec{\chi}\right)=\int\left|f\left(\overrightarrow{\mathrm{k}}_{i} ; \overrightarrow{\mathrm{n}} ; \vec{\chi}\right)\right|^{2} d \Omega_{\overrightarrow{\mathrm{n}}}
$$

is the conventional spheroidal cross section. In practice, the cross sections in Eq. (4.2) are not experimentally observed quantities. For comparison with experiment we have to take the average over the orientation $\vec{\chi}$ and obtain the mean total cross sections

$$
\begin{aligned}
\bar{\Sigma}\left(\overrightarrow{\mathrm{k}}_{1}, \overrightarrow{\mathrm{k}}_{2}\right)= & \bar{\sigma}(k)+\left(1+|a|^{2}\right)^{-1} \\
& \times \operatorname{Re}\left(\frac{a}{i k} \int\left[f\left(\overrightarrow{\mathrm{k}}_{2} ; \overrightarrow{\mathrm{n}}_{1} ; \vec{\chi}\right)-f^{*}\left(\overrightarrow{\mathrm{k}}_{1} ; \overrightarrow{\mathrm{n}}_{2} ; \vec{\chi}\right)\right] d \Omega \vec{\chi}\right),
\end{aligned}
$$

where

$$
\bar{\Sigma}\left(\overrightarrow{\mathrm{k}}_{1}, \overrightarrow{\mathrm{k}}_{2}\right)=\frac{1}{4 \pi} \int \Sigma\left(\overrightarrow{\mathrm{k}}_{1}, \overrightarrow{\mathrm{k}}_{2} ; \vec{\chi}\right) d \Omega_{\vec{\chi}}
$$

and

$$
\bar{\sigma}(k)=\frac{1}{4 \pi} \int \sigma\left(\overrightarrow{\mathrm{k}}_{i} ; \vec{\chi}\right) d \Omega \overrightarrow{\mathrm{\chi}} .
$$


After the average, the mean scattering amplitude and the mean differential cross section depend simply on the relative angle between the incident and scattered directions,

$$
\bar{f}_{k}\left(\overrightarrow{\mathrm{n}}_{i} \cdot \overrightarrow{\mathrm{n}}\right)=\frac{1}{4 \pi} \int f\left(\overrightarrow{\mathrm{k}}_{i} ; \overrightarrow{\mathrm{n}} ; \vec{\chi}\right) d \Omega \overrightarrow{\mathrm{x}}
$$

and

$$
d \bar{\sigma}_{k}\left(\overrightarrow{\mathrm{n}}_{i} \cdot \overrightarrow{\mathrm{n}}\right)=\frac{1}{4 \pi} d \Omega_{\overrightarrow{\mathrm{n}}} \int\left|f\left(\overrightarrow{\mathrm{k}}_{i} ; \overrightarrow{\mathrm{n}} ; \vec{\chi}\right)\right|^{2} d \Omega_{\vec{\chi}} .
$$

This dependence comes from the requirement that the average mean physical quantities are independent of the choice of the describing coordinate systems. From Eq. (4.6), we may rewrite Eq. (4.4) as

$$
\begin{aligned}
\bar{\Sigma}\left(\overrightarrow{\mathrm{k}}_{1}, \overrightarrow{\mathrm{k}}_{2}\right)= & \bar{\sigma}(k)+\frac{8 \pi}{k}\left(1+|a|^{2}\right)^{-1} \\
& \times \operatorname{Re} a \operatorname{Im} \bar{f}_{k}\left(\overrightarrow{\mathrm{n}}_{1} \cdot \overrightarrow{\mathrm{n}}_{2}\right) .
\end{aligned}
$$

Equation (3.3) and (4.8) are similar. To determine the beam-dependent factor $a$ in Eq. (4.8), we replace the spheroidal scatterer by a spherical scatterer, and measure the spherical differential cross sections. From Eq. (3.4) one obtains

$$
\begin{aligned}
\operatorname{Im} \bar{f}_{k}\left(\overrightarrow{\mathrm{n}}_{1} \cdot \overrightarrow{\mathrm{n}}_{2}\right)= & \frac{k}{4 \pi}\left[\bar{\Sigma}\left(\overrightarrow{\mathrm{k}}_{1}, \overrightarrow{\mathrm{k}}_{2}\right)-\bar{\sigma}(k)\right] \\
& \times\left(\frac{d \Sigma\left(\overrightarrow{\mathrm{k}}_{1}, \overrightarrow{\mathrm{k}}_{2}, \overrightarrow{\mathrm{n}}\right)}{d \sigma_{k}\left(\overrightarrow{\mathrm{n}}_{i} \cdot \overrightarrow{\mathrm{n}}\right)}-1\right)^{-1},
\end{aligned}
$$

where $\vec{n}_{1} \cdot \vec{n}=\vec{n}_{2} \cdot \vec{n}$. The equation expresses the fact that the mean imaginary part of the spheroidal scattering amplitude can be experimentally measured. In theory this is more easy to handle than the mean differential cross section. We shall proceed further.

For scattering by a spheroidal potential, ${ }^{9}$ the spheroidal analysis is used and the scattering amplitude is expressed as

$$
\begin{aligned}
f\left(\overrightarrow{\mathrm{k}}_{i} ; \overrightarrow{\mathrm{n}} ; \vec{\chi}\right)= & \frac{1}{i k} \sum_{n=0}^{\infty} \sum_{m=-\bar{n}}^{\bar{n}} N_{m}^{-1} \bar{n}(c) S_{m \bar{n}}\left(c, \eta_{i}\right) \\
& \times S_{m \bar{n}}(c, \eta)\left(e^{2 i \delta_{m}-\bar{n}(k)}-1\right) e^{i m\left(\phi-\phi_{i}\right)},
\end{aligned}
$$

where $\eta_{i}=\overrightarrow{\mathrm{n}}_{i} \cdot \vec{\chi}$ and $\eta=\overrightarrow{\mathrm{n}} \cdot \vec{\chi}$. The spheroidal coordinate system is chosen such that the $z$ axis coincides with the orientation vector $\vec{\chi} . \phi$ and $\phi_{i}$ are the azimuthal angles of vectors $\vec{n}$ and $\vec{n}_{i}$. The constant $c\left(c=\frac{1}{2} k d\right)$ is related to the interfocal distance $d$ of the spheroidal coordinates. The physical meaning of spheroidal phase shifts $\delta_{m n}(k)$ is similar to that of spherical phase shifts $\delta_{l}(k)$. The spheroidal angle function $S_{m n}(c, \eta)$ has a normalization constant $N_{m n}(c)$,

$$
\int_{-1}^{1} S_{m n}(c, \eta) S_{m n^{\prime}}(c, \eta) d \eta=\delta_{n n^{\prime}} N_{m n}(c),
$$

and can be expanded in terms of the associated Legendre functions

$$
S_{m n}(c, \eta)=\sum_{r=0,1}^{\infty} d_{r}^{m n} P_{m+r}^{m}(\eta)
$$

$\sum$ 'denotes the summation in an even or odd value of $r$, depending upon whether $n-m$ is even or odd. The expansion coefficient $d_{r}^{m n}$ can be found in Flammer's book. ${ }^{10}$ The mean scattering amplitude has the form

$$
\begin{aligned}
\vec{f}_{k}\left(\overrightarrow{\mathrm{n}}_{i} \cdot \overrightarrow{\mathrm{n}}\right) & =\frac{1}{4 \pi} \int f\left(\overrightarrow{\mathrm{k}}_{i} ; \overrightarrow{\mathrm{n}} ; \vec{\chi}\right) d S \vec{\chi}_{\overline{\mathrm{x}}} \\
& =\frac{1}{i k} \sum_{n=0}^{\infty} \sum_{m=-n}^{n} \tilde{S}_{m n}\left(c, \overrightarrow{\mathrm{n}}_{i} \cdot \overrightarrow{\mathrm{n}}\right)\left(e^{2 i \delta_{m n}(k)}-1\right),
\end{aligned}
$$

where

$$
\begin{aligned}
\tilde{S}_{m \bar{n}}\left(c, \overrightarrow{\mathrm{n}}_{i} \cdot \overrightarrow{\mathrm{n}}\right)= & \frac{1}{4 \pi N_{m \bar{n}}(c)} \int S_{m \vec{n}}\left(c, \eta_{i}\right) S_{m \bar{n}}(c, \eta) \\
& \times e^{i m\left(\phi-\phi_{i}\right)} d \Omega_{\overrightarrow{\mathrm{x}}} .
\end{aligned}
$$

The integration in Eq. (4.14) is rotationally invariant and depends on the relative angle between vectors $\vec{n}$ and $\vec{n}_{i}$. To evaluate the integration, ${ }^{11}$ we use the expansion

$$
\tilde{S}_{m \bar{n}}\left(c, \overrightarrow{\mathrm{n}}_{i} \cdot \overrightarrow{\mathrm{n}}\right)=\sum_{l=0}^{\infty} b_{l}^{m \bar{n}} \frac{2 l+1}{4 \pi} P_{l}\left(\overrightarrow{\mathrm{n}}_{i} \cdot \overrightarrow{\mathrm{n}}\right),
$$

where

$$
\begin{aligned}
b_{l}^{m n} & =\int \tilde{S}_{m \bar{n}}\left(c, \overrightarrow{\mathrm{n}}_{i} \cdot \overrightarrow{\mathrm{n}}\right) P_{l}\left(\overrightarrow{\mathrm{n}}_{i} \cdot \overrightarrow{\mathrm{n}}\right) d \Omega_{\overrightarrow{\mathrm{n}}_{i}} \\
& =\frac{1}{4 \pi} \iint \tilde{S}_{m \bar{n}}\left(c, \overrightarrow{\mathrm{n}}_{i} \cdot \overrightarrow{\mathrm{n}}\right) P_{l}\left(\overrightarrow{\mathrm{n}}_{i} \cdot \overrightarrow{\mathrm{n}}\right) d \Omega_{\overrightarrow{\mathrm{n}}_{i}} d \Omega_{\overrightarrow{\mathrm{n}}} .
\end{aligned}
$$

Through the utilization of the addition theorem Eq. (3.9) and the orthogonality relations between the spherical harmonics, we might carry out the above integration with the help of Eqs. (4.12) and (4.14):

$$
b_{l}^{m n}=\frac{4 \pi}{(2 l+1)^{2}} N_{m n}^{-1}(c)\left(d_{l-m}^{m n}\right)^{2} \frac{(l+|m|) !}{(l-|m|) !} .
$$

The function $\tilde{S}_{m n}\left(c, \vec{n}_{i} \cdot \overrightarrow{\mathrm{n}}\right)$ is real, which follows 
directly from Eqs. (4.15) and (4.17). The experimentally measurable quantity in Eq. (4.9), which is the imaginary part of the mean scattering amplitude in Eq. (4.15), has the expression

$$
\operatorname{Im} \bar{f}_{k}\left(\overrightarrow{\mathrm{n}}_{i} \cdot \overrightarrow{\mathrm{n}}\right)=\frac{2}{k} \sum_{n=0}^{\infty} \sum_{m=-\bar{n}}^{\bar{n}} \tilde{S}_{m \bar{n}}\left(c, \overrightarrow{\mathrm{n}}_{i} \cdot \overrightarrow{\mathrm{n}}\right) \sin ^{2} \delta_{m \bar{n}}(k) .
$$

We may express the conventional mean differential cross section in terms of the spheroidal phase shifts. This expression contains at least four summations and is more tedious than Eq. (4.18). Hence the observed mean imaginary part of the scattering amplitude is related to the spheroidal phase shifts in a more straightforward manner than the conventional mean differential cross section. In a coherent scattering we may observe the coherent differential cross section. This cross section contains a term

$$
\operatorname{Re}\left(a \int f\left(\overrightarrow{\mathrm{n}}_{1} ; \overrightarrow{\mathrm{n}} ; \overrightarrow{\mathrm{\chi}}\right) f *\left(\overrightarrow{\mathrm{n}}_{2} ; \overrightarrow{\mathrm{n}} ; \vec{\chi}\right) d \Omega \overrightarrow{\mathrm{x}}\right),
$$

which denotes the correlation of the scattering amplitude at different angles. Now it should be clear to us that the coherent scattering provides experimentally more-measurable quantities, and yields more direct information than the conventional scattering.

\section{SPIN-DEPENDENT POTENTIAL}

Hitherto we have considered only the coherent scattering of particles by a fixed potential, which does not depend on their spins. Under this condition the spins do not affect the scattering process at all. Let us now examine the coherent scattering, where the potential depends' significantly on their spins. Let us restrict ourselves to the simplest case, where the particles in the incident beams have spin- $-\frac{1}{2}$ and the potential has the form

$$
V(r)=V_{1}(r)+(\vec{\sigma} \cdot \vec{L}) V_{2}(r) \text {. }
$$

The potentials $V_{1}(r)$ and $V_{2}(r)$ are spherically symmetric. $\vec{\sigma}$ is the Pauli spin operator and $\overrightarrow{\mathrm{L}}$ the orbital angular momentum. In the case of electrons, $V_{1}(r)$ and $V_{2}(r)$ are related, but we shall not make this assumption. The two coherent beams are different in directions but the same in energy. For the spin polarizations, we do not know whether they are the same or different in the coherent beams. To be general, we consider they might be different. Now the incident beams are described by a pure-state vector

$$
e^{i \vec{k}_{1} \cdot \vec{r}}\left|\chi_{i}^{1}\right\rangle+a e^{i \vec{k}_{2} \cdot \vec{r}}\left|\chi_{i}^{2}\right\rangle
$$

The spinors $\left|\chi_{i}^{1}\right\rangle$ and $\left|\chi_{i}^{2}\right\rangle$ denote the spin polarizations. The scattered wave with the final spin polarization $\chi_{f}$ satisfies the Schrödinger equation

$$
\begin{aligned}
-\frac{\hbar^{2}}{2 m} \nabla^{2} \psi\left(\overrightarrow{\mathrm{r}} ; \chi_{f} ; \chi_{i}\right) & +\sum_{h}\left\langle\chi_{f}\left|V_{1}(r)+(\vec{\sigma} \cdot \overrightarrow{\mathrm{L}}) V_{2}(r)\right| \chi_{h}\right\rangle \\
& \times \psi\left(\overrightarrow{\mathrm{r}} ; \chi_{h}, \chi_{i}\right)=E \psi\left(\overrightarrow{\mathrm{r}} ; \chi_{f}, \chi_{i}\right),
\end{aligned}
$$

and the asymptotic condition

$$
\begin{aligned}
\psi\left(\overrightarrow{\mathrm{r}} ; \chi_{f} ; \chi_{i}\right) \underset{r \rightarrow \infty}{\sim} e^{i \overrightarrow{\mathrm{k}}_{1} \cdot \overrightarrow{\mathrm{r}}}\left\langle\chi_{f} \mid \chi_{i}^{1}\right\rangle+a e^{i \overrightarrow{\mathrm{k}}_{2} \cdot \overrightarrow{\mathrm{r}}}\left\langle\chi_{f} \mid \chi_{i}^{2}\right\rangle \\
+F\left(\overrightarrow{\mathrm{k}}_{1}, \overrightarrow{\mathrm{k}}_{2} ; \overrightarrow{\mathrm{n}}^{\prime} \chi_{f} ; \chi_{i}^{1}, \chi_{i}^{2}\right) e^{i k r} / r,(5.4)
\end{aligned}
$$

The Schrơdinger equation (5.3) and the incident wave in Eq. (5.4) are linear. By the same token, as we have discussed in Sec. II for the spin-independent case, the coherent-scattering amplitude $F\left(\overrightarrow{\mathrm{k}}_{1}, \overrightarrow{\mathrm{k}}_{2} ; \overrightarrow{\mathrm{n}} ; \chi_{f} ; \chi_{i}^{1} ; \chi_{i}^{2}\right)$ in Eq. (5.4) can be expressed as the sum of the two conventional scattering amplitudes,

$$
\begin{aligned}
F\left(\overrightarrow{\mathrm{k}}_{1}, \overrightarrow{\mathrm{k}}_{2} ; \overrightarrow{\mathrm{n}} ; \chi_{f} ; \chi_{i}^{1} ; \chi_{i}^{2}\right)= & f\left(\overrightarrow{\mathrm{k}}_{1} ; \overrightarrow{\mathrm{n}} ; \chi_{f} ; \chi_{i}^{1}\right) \\
& +a f\left(\overrightarrow{\mathrm{k}}_{2} ; \overrightarrow{\mathrm{n}} ; \chi_{f} ; \chi_{i}^{2}\right) .
\end{aligned}
$$

This relation is equivalent to the one in Eq. (2.7). It is customary ${ }^{6}$ to write the scattering amplitude in the form

$$
\begin{aligned}
f\left(\overrightarrow{\mathrm{k}}_{j} ; \overrightarrow{\mathrm{n}} ; \chi_{f} ; \chi_{i}\right)= & \left\langle\chi_{f}\right| f_{k}\left(\overrightarrow{\mathrm{n}}_{j} \cdot \overrightarrow{\mathrm{n}}\right)+i \overrightarrow{\mathrm{\sigma}} \cdot\left(\overrightarrow{\mathrm{n}}_{j} \times \overrightarrow{\mathrm{n}}\right) \\
& \times g_{k}\left(\overrightarrow{\mathrm{n}}_{j} \cdot \overrightarrow{\mathrm{n}}\right)\left|\chi_{i}\right\rangle .
\end{aligned}
$$

From the above two equations we rewrite the coherent-scattering amplitude as

$$
F\left(\overrightarrow{\mathrm{k}}_{1}, \overrightarrow{\mathrm{k}}_{2} ; \overrightarrow{\mathrm{n}}_{;} \chi_{f} ; \chi_{i}^{1} ; \chi_{i}^{2}\right)=\left\langle\chi_{f}\left|f_{k}\left(\overrightarrow{\mathrm{n}}_{1} \cdot \overrightarrow{\mathrm{n}}\right)+i \vec{\sigma} \cdot\left(\overrightarrow{\mathrm{n}}_{1} \times \overrightarrow{\mathrm{n}}\right) g_{k}\left(\overrightarrow{\mathrm{n}}_{1} \cdot \overrightarrow{\mathrm{n}}\right)\right| \chi_{i}^{1}\right\rangle+a\left\langle\chi_{f}\left|f_{k}\left(\overrightarrow{\mathrm{n}}_{2} \cdot \overrightarrow{\mathrm{n}}\right)+i \vec{\sigma} \cdot\left(\overrightarrow{\mathrm{n}}_{2} \times \overrightarrow{\mathrm{n}}\right) g_{k}\left(\overrightarrow{\mathrm{n}}_{2} \cdot \overrightarrow{\mathrm{n}}\right)\right| \chi_{i}^{2}\right\rangle
$$

The coherent differential cross section is

$$
\begin{aligned}
d \Sigma\left(\overrightarrow{\mathrm{k}}_{1}, \overrightarrow{\mathrm{k}}_{2} ; \overrightarrow{\mathrm{n}} ; \chi_{f} ; \chi_{i}^{1} ; \chi_{i}^{2}\right)=\left(1+|a|^{2}\right)^{-1}\left|F\left(\overrightarrow{\mathrm{k}}_{1}, \overrightarrow{\mathrm{k}}_{2} ; \overrightarrow{\mathrm{n}} ; \chi_{f} ; \chi_{i}^{1} ; \chi_{i}^{2}\right)\right|^{2} d \Omega \overrightarrow{\mathrm{n}} & =\left(1+|a|^{2}\right)^{-1}\left\{\left|f\left(\overrightarrow{\mathrm{k}_{1}} ; \overrightarrow{\mathrm{n}} ; \chi_{f} ; \chi_{i}^{1}\right)\right|^{2}+\left|a f\left(\overrightarrow{\mathrm{k}}_{2} ; \overrightarrow{\mathrm{n}} ; \chi_{f} ; \chi_{i}^{2}\right)\right|^{2}\right. \\
& \left.+2 \operatorname{Re}\left[a f\left(\overrightarrow{\mathrm{k}}_{2} ; \overrightarrow{\mathrm{n}} ; \chi_{f} ; \chi_{i}^{2}\right) f^{*}\left(\overrightarrow{\mathrm{k}}_{1} ; \overrightarrow{\mathrm{n}} ; \chi_{f} ; \chi_{i}^{1}\right)\right]\right\} d \Omega \overrightarrow{\mathrm{n}} .
\end{aligned}
$$

If the final spin polarization is not observed, the coherent cross section has the form 


$$
\begin{gathered}
\bar{\Sigma}\left(\overrightarrow{\mathrm{k}}_{1}, \overrightarrow{\mathrm{k}}_{2} ; \chi_{i}^{1} ; \chi_{i}^{2}\right)=\sum_{f} \int d \Sigma\left(\overrightarrow{\mathrm{k}}_{1}, \overrightarrow{\mathrm{k}}_{2} ; \overrightarrow{\mathrm{n}} ; \chi_{f} ; \chi_{i}^{1} ; \chi_{i}^{2}\right)=\left(1+|a|^{2}\right)^{-1}\left[\sum_{f} 2 \operatorname{Re}\left(\int a f\left(\overrightarrow{\mathrm{k}}_{2} ; \overrightarrow{\mathrm{n}} ; \chi_{f} ; \chi_{i}^{2}\right) f *\left(\overrightarrow{\mathrm{k}}_{1} ; \overrightarrow{\mathrm{n}} ; \chi_{f} ; \chi_{i}^{1}\right)\right) d \Omega_{\overrightarrow{\mathrm{n}}}\right. \\
\left.+\bar{\sigma}\left(k, \chi_{i}^{1}\right)+|a|^{2} \bar{\sigma}\left(k, \chi_{i}^{2}\right)\right],
\end{gathered}
$$

where

$$
\begin{aligned}
\bar{\sigma}\left(k, \chi_{i}^{\alpha}\right)=\sum_{f} \int\left|f\left(\overrightarrow{\mathrm{n}}_{j}, \overrightarrow{\mathrm{n}} ; \chi_{f} ; \chi_{i}^{\alpha}\right)\right|^{2} d \Omega_{\overrightarrow{\mathrm{n}}}=\int & {\left[\left|f_{k}\left(\overrightarrow{\mathrm{n}}_{j} \cdot \overrightarrow{\mathrm{n}}\right)\right|^{2}+\left|\overrightarrow{\mathrm{n}}_{j} \times \overrightarrow{\mathrm{n}}\right|^{2}\left|g_{k}\left(\overrightarrow{\mathrm{n}}_{j} \cdot \overrightarrow{\mathrm{n}}\right)\right|^{2}+2 \operatorname{Re}\left\langle\chi_{i}^{\alpha}\right| i \vec{\sigma} \cdot\left(\overrightarrow{\mathrm{n}_{j}} \times \overrightarrow{\mathrm{n}}\right) g_{k}\left(\overrightarrow{\mathrm{n}}_{j} \cdot \overrightarrow{\mathrm{n}}\right)\right.} \\
& \left.\times f_{\vec{k}}^{*}\left(\overrightarrow{\mathrm{n}}_{j} \cdot \overrightarrow{\mathrm{n}}\right)\left|\chi_{i}^{\alpha}\right\rangle\right] d \Omega_{\overrightarrow{\mathrm{n}}}
\end{aligned}
$$

is the conventional elastic cross section. For elastic scattering the conservation of the number of the particles leads to a similar restriction ${ }^{6}$ as in Eq. (2.10):

$$
\begin{aligned}
& f\left(\overrightarrow{\mathrm{k}}_{j} ; \overrightarrow{\mathrm{n}} ; \chi_{f} ; \chi_{i}^{j}\right)-f^{*}\left(\overrightarrow{\mathrm{k}} ; \overrightarrow{\mathrm{n}}_{j} ; \chi_{f} ; \chi_{i}^{j}\right) \\
& \quad=\frac{i k}{2 \pi} \sum_{h} \int f\left(\overrightarrow{\mathrm{k}}_{j} ; \overrightarrow{\mathrm{n}}^{\prime} ; \chi_{h} ; \chi_{i}^{j}\right) f^{*}\left(\overrightarrow{\mathrm{k}} ; \overrightarrow{\mathrm{n}}^{\prime} ; \chi_{\boldsymbol{h}} ; \chi_{f}\right) d \Omega \overrightarrow{\mathrm{n}}^{\prime},
\end{aligned}
$$

where $\overrightarrow{\mathrm{k}}=k \overrightarrow{\mathrm{n}}$ is the momentum of the beam in the direction of detection. With the help of the above equation we may rewrite Eq. (5.9) as

$$
\begin{aligned}
\Sigma\left(\overrightarrow{\mathrm{k}}_{1}, \overrightarrow{\mathrm{k}}_{2} ; \chi_{i}^{1} ; \chi_{i}^{2}\right)= & \left(1+|a|^{2}\right)^{-1} \\
\times[ & (8 \pi / k) \operatorname{Re} a \operatorname{Im} f\left(\overrightarrow{\mathrm{k}}_{1}, \overrightarrow{\mathrm{n}}_{2} ; \chi_{i}^{2} ; \chi_{i}^{1}\right) \\
& \left.+\bar{\sigma}\left(k, \chi_{i}^{1}\right)+|a|^{2} \bar{\sigma}\left(k, \chi_{i}^{2}\right)\right] .
\end{aligned}
$$

This expression is similar to the one in Eq. (3.3), which expresses the coherent-cross-section dependence on the imaginary part of the scattering amplitude in the absence of spin. By measuring the coherent and conventional cross sections along with the relative factor $a$ of the coherent beams, this imaginary part can be experimentally determined. We would like to discuss the structure of this imaginary part. After that, the measurement of the relative factor $a$ is outlined.

If in the coherent beams the spin polarizations are the same, $\left\langle\chi_{i}^{2} \mid \chi_{i}^{1}\right\rangle=1$, then in terms of the customary notation in Eq. (5.6) we obtain

$$
\begin{aligned}
\operatorname{Im} f\left(\overrightarrow{\mathrm{k}}_{1}, \overrightarrow{\mathrm{n}}_{2} ; \chi_{i}^{j} ; \chi_{i}^{j}\right)= & \operatorname{Im} f_{k}\left(\overrightarrow{\mathrm{n}}_{1} \cdot \overrightarrow{\mathrm{n}}_{2}\right) \\
& -(-)^{j}\left|\overrightarrow{\mathrm{n}}_{1} \times \overrightarrow{\mathrm{n}}\right| \operatorname{Reg}_{k}\left(\overrightarrow{\mathrm{n}}_{1} \circ \overrightarrow{\mathrm{n}}_{2}\right) .
\end{aligned}
$$

Here we have supposed that the axis of spin quantization is parallel to $\overrightarrow{\mathrm{n}}_{1} \times \overrightarrow{\mathrm{n}}_{2}$, and $j=1,2$ means spin up and down, respectively. Furthermore if the initial beams are not polarized, the second term will drop out. Then through the use of the coherent polarized beam the imaginary part $f_{k}\left(\vec{n}_{1} \cdot \vec{n}_{2}\right)$ and real part $g_{k}\left(\vec{n}_{1} \cdot \vec{n}_{2}\right)$ are determined directly and separately. On the other hand, the nonpolarized coherent beam determines only the imaginary part $f_{k}\left(\overrightarrow{\mathrm{n}}_{1} \cdot \overrightarrow{\mathrm{n}}_{2}\right)$.

If in the coherent beams the spin polarization are opposite, $\left\langle\chi_{i}^{2} \mid \chi_{i}^{1}\right\rangle=0$. These beams do not create any direct-interference phenomenon. In a sense, they are actually incoherent. Through a direct verification, we get

$$
\operatorname{Im} f\left(\overrightarrow{\mathrm{k}}_{1}, \overrightarrow{\mathrm{n}}_{2} ; \chi_{i}^{2} ; \chi_{i}^{1}\right)=0 \text { for }\left\langle\chi_{i}^{2} \mid \chi_{i}^{1}\right\rangle=0 .
$$

The measurement of the relative factor $a$ can be accomplished much ir the same way as we have done in the spin-independent case. If the spin polarizations are not observed before and after scattering, the coherent differential cross section in Eq. (5.8) in terms of the customary notation as in Eq. (5.6) can be written

$$
\begin{aligned}
d \Sigma\left(\overrightarrow{\mathrm{k}}_{1}, \overrightarrow{\mathrm{k}}_{2} ; \overrightarrow{\mathrm{n}}\right)=\frac{1}{2} \sum_{j} \sum_{f} d \Sigma\left(\overrightarrow{\mathrm{k}}_{1}, \overrightarrow{\mathrm{k}}_{2}, \overrightarrow{\mathrm{n}} ; \chi_{f} ; \chi_{i}^{j} ; \chi_{i}^{j}\right)= & \left(1+|a|^{2}\right)^{-1}\left\{d \bar{\sigma}_{k}\left(\overrightarrow{\mathrm{n}}_{1} \cdot \overrightarrow{\mathrm{n}}\right)+|a|^{2} d \bar{\sigma}_{k}\left(\overrightarrow{\mathrm{n}}_{2} \cdot \overrightarrow{\mathrm{n}}\right)+2 \operatorname{Re}\left[a\left(f \overrightarrow{\mathrm{n}}_{1} \cdot \overrightarrow{\mathrm{n}}\right) f_{k}\left(\overrightarrow{\mathrm{n}}_{2} \cdot \overrightarrow{\mathrm{n}}\right)\right.\right. \\
& \left.\left.\left.+\left(\overrightarrow{\mathrm{n}}_{1} \times \overrightarrow{\mathrm{n}}\right) \cdot\left(\overrightarrow{\mathrm{n}}_{2} \times \overrightarrow{\mathrm{n}}\right) g_{k}^{*}\left(\overrightarrow{\mathrm{n}}_{1} \cdot \overrightarrow{\mathrm{n}}\right) g_{k}\left(\overrightarrow{\mathrm{n}}_{2} \cdot \overrightarrow{\mathrm{n}}\right)\right)\right] d \Omega \overrightarrow{\mathrm{n}}\right\}
\end{aligned}
$$

where

$$
d \bar{\sigma}_{k}\left(\overrightarrow{\mathrm{n}}_{j} \cdot \overrightarrow{\mathrm{n}}\right)=\left|f_{k}\left(\overrightarrow{\mathrm{n}}_{j} \cdot \overrightarrow{\mathrm{n}}\right)\right|^{2}+\left|\overrightarrow{\mathrm{n}}_{j} \times \overrightarrow{\mathrm{n}}\right|^{2}\left|g_{k}\left(\overrightarrow{\mathrm{n}}_{j} \cdot \overrightarrow{\mathrm{n}}\right)\right|^{2}
$$

is the unpolarized differential cross section in the conventional scattering. Let us take $\vec{n}_{1} \cdot \vec{n}=\vec{n}_{2} \cdot \vec{n}$, 
then

$$
\begin{aligned}
d \bar{\Sigma}\left(\overrightarrow{\mathrm{k}}_{1}, \overrightarrow{\mathrm{k}}_{2} ; \overrightarrow{\mathrm{n}}\right)= & \left(1+\frac{2 \operatorname{Re} a}{1+|a|^{2}}\right) d \bar{\sigma}_{k}\left(\overrightarrow{\mathrm{n}}_{1} \cdot \overrightarrow{\mathrm{n}}\right) \\
& +\frac{2 \operatorname{Re} a}{1+|a|^{2}}\left[\left(\overrightarrow{\mathrm{n}}_{1} \cdot \overrightarrow{\mathrm{n}}_{2}\right)-1\right]\left|g_{k}\left(\overrightarrow{\mathrm{n}}_{1} \cdot \overrightarrow{\mathrm{n}}\right)\right|^{2} d \Omega \overrightarrow{\mathrm{n}} .
\end{aligned}
$$

The factor $\left|g_{k}\left(\overrightarrow{\mathrm{n}}_{1} \cdot \overrightarrow{\mathrm{n}}\right)\right|^{2}$ can be measured in the conventional scattering of a polarized beam and is related to the spin-flip differential cross section. Hence in the coherent scattering as a rule, we are not only able to measure the imaginary part of the scattering amplitude, but also the relative strength and phase of the coherent beams.

\section{COMMENTS}

We have presented a theory, which exhibits the characteristic features of scatterings initiated by two coherent beams. It reveals that the imaginary part of the scattering amplitude is an experimentally measurable quantity. To know the imaginary part of the amplitude is quite important for certain aspects of quantum mechanics, for example, the dispersion relation for fixed momentum transfer. This relation has not yet been experimentally verified. Near the resonance region the scattering amplitude takes a certain form, which is usually described by a Breit-Wigner formula. Knowledge of the imaginary part of the amplitude would yield a detailed knowledge of resonances. For the phase shifts, it will do much the same. In this way, the scattering initiated by coherent beams provides us new tools for the understanding of molecular and nuclear physics.

The proposed scattering is also important for the experimental study of diatomic molecules and deformed nuclei. in low-energy electron-molecule scattering differential cross sections are difficult to obtain and their measurements are still fragmentary. ${ }^{7}$ On the other hand, the total cross section is easy to measure. To determine the mean imaginary part of the spheroidal scattering amplitude we only need to measure the coherent and conventional total cross sections, as in Eq. (4.8). The factor $a$ describes the coherent beams. It can be measured in other ways, not necessarily through the method proposed in Eq. (4.9), which we will discuss in a later publication.

At present, experimentally obtained coherent electron beams are used for direct-interference experiments, in which interference fringes are observed. The proposed scattering method yields a new usage for coherent electron beams and will stimulate experimental work in a field which has been inactive in the past decades. ${ }^{12}$

\section{ACKNOWLEDGMENTS}

In conclusion, the author wishes to express his appreciation to Professor S. P. Almeida and Professor D. Kaplan for interesting conversations on several matters presented here.
${ }^{1}$ M. Born and E. Wolf, Principles of Optics (Pergamon, New York, 1965).

${ }^{2}$ L. Marton, Phys. Rev. 85, 1057 (1952); L. Marton, J. A. Simpson, and J. A. Suddeth, Phys. Rev. 90,490 (1953); Rev. Sci. Instrum. 25, 1099 (1954); J. A. Simpson, Rev. Sci. Instrum. 25, 1105 (1954); L. Marton, Science 118,470 (1953).

${ }^{3}$ G. Mollenstedt and H. Duker, Z. Naturforsch. A $\underline{8}, 79$ (1953); Naturwissenshaften 42 , 41 (1954); H. Duker, Z. Naturforsch. A $10,256(1955)$.

${ }^{4}$ J. A. Simpson, Rev. Mod. Phys. 28, 254 (1956).

${ }^{5}$ D. Gabor, Rev. Mod. Phys. 28,261 (1956).

${ }^{6}$ L. D. Landau and E. M. Lifshitz, Quantum Mechanics (Pergamon, New York, 1965).

${ }^{7}$ D. E. Golden, N. F. Lane, A. Temlein and E. Gerjuoy,
Rev. Mod. Phys. $\underline{43}, 642$ (1971).

${ }^{8}$ H. Stier, Z. Phys. 76 , 439 (1952); J. B. Fisk, Phys. Rev. 49, 167 (1936); K. Takayanagi, Prog. Theor. Phys. Suppl. 40,216 (1967); J. Rainwater, Phys. Rev. 79, 432 (1950); S. Granger and R. D. Spence, Phys. Rev. $\underline{83}, 460$ (1951); Ming Chiang Li, Phys. Rev. D 1,326 (1970).

${ }^{9}$ Ming Chiang Li, J. Math. Phys. 12 , 936 (1971); J. Math. Phys. 13, 1381 (1972); J. Math. Phys. 14, 1358 (1973); J. Math. Phys. (to be published).

${ }^{10} \mathrm{C}$. Flammer, Spheroidal Wave Functions (Stanford

U. P., Palo Alto, Calif., 1957).

${ }^{11} \mathrm{~K}$. Takayanagi and Y. Itikawa, J. Phys. Soc. Jap. $\underline{24}$, 160 (19ûs).

${ }^{12} \mathrm{~J}$. A. Simpson (private communication). 